\title{
Analysis of the primary and post-treatment antibiotic resistance of Helicobacter pylori in Nanjing area
}

jiang zongdan ( $\sim$ jzongdan@sina.com )

Nanjing First Hospital https://orcid.org/0000-0001-7881-4424

Bang-Shun He

Nanjing First Hospital

Zhen-Yu Zhang

Nanjing First Hospital

Shu-Kui Wang

Nanjing First Hospital

Dan Ran

Nanjing First Hospital

Zhi-Bing WANG

Nanjing First Hospital

Research

Keywords: Helicobacter pylori, The primary and post-treatment antibiotic resistance, Nanjing

Posted Date: January 8th, 2020

DOl: https://doi.org/10.21203/rs.2.20406/v1

License: (9) (i) This work is licensed under a Creative Commons Attribution 4.0 International License.

Read Full License 


\section{Abstract}

Background: To investigate the current status and trend of Helicobacter pylori H. pyloriखantibiotics resistance over 5 years in Nanjing area $\$ Then we evaluated the primary and post-treatment antibiotic resistance of $H$. pylori in Nanjing area.

Methods: During the period from July 2017 to December 2019, 1533 gastric mucosal specimens from patients with positive $H$. pylori confirmed by breath test or rapid urease test were collected for isolation and identify $H$. pylori. The agar dilution method was used for antibiotic resistance test. The antibiotic resistance of $H$. pylori were compared with that in the period from May 2014 to May 2015.

Results: The result showed that the resistance rates of $\mathrm{H}$. pylori to amoxicillin, clarithromycin, levofloxacin, furazolidone, tetracycline and metronidazole were $2.74 \%, 47.03 \%, 33.59 \%, 0.91 \%, 0.52 \%$ and $80.76 \%$, respectively in the period of July 2017 to December 2019. Compared with the period of May 2014 to May 2015 , the resistance rate of metronidazole was the most obvious, from $60.5 \%$ to $80.76 \%$ in the period of July 2017 to December 2019, followed by clarithromycin from $29.5 \%$ to $47.03 \%$, and levofloxacin from $14.7 \%$ to $33.59 \%$. The resistance rates of $H$. pylori (primary Vs post-treatment) to amoxicillin, clarithromycin, levofloxacin, furazolidone, tetracycline and metronidazole were $1.83 \% \mathrm{Vs}$ $6.08 \%, 38.62 \%$ Vs $77.81 \%, 27.41 \%$ Vs $56.23 \%, 0.58 \%$ Vs $2.13 \%, 0.33 \%$ Vs $1.22 \%, 78.57 \%$ Vs $88.75 \%$, respectively during the period of July 2017 to December 2019.

Conclusions: Antibiotic resistance of $H$. pylori remained a problem for the effective eradication of this pathogen and its associated diseases in Nanjing area. For post-treatment eradication patients, clinicians should took into account regional antibiotic resistance rate, personal antibiotic exposure history,

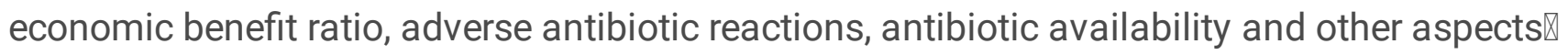

\section{Background}

Helicobacter pylori(H. Pylori) is the major cause of chronic gastritis, peptic ulcers, gastric adenocarcinoma, gastric mucosa-associated lymphoid tissue (MALT) lymphoma, and various other digestive system diseases [1-3]. Eradicating the pathogen is crucial for the prevention and treatment of these diseases [4].

The eradication of $\mathrm{H}$. Pylori is an important issue in the field of preventive medicine. The Maastricht $\mathrm{V}$ consensus Report has recommended that the standard triple therapy should now be avoided in areas where clarithromycin resistance is high (>15\%) [5]. China has a large H. Pylori infected population that exhibits complicated antibiotic resistance features [6]. Several studies have reported antibiotic resistance in some regions of China. For example, 20 to $50 \%$ of cases are resistant to clarithromycin [7-8]. The resistance rate to metronidazole in the southeast coastal province of China was 95.4\% [9]. With such high resistance rates, the eradication rate of the standard triple therapy for $\mathrm{H}$. Pylori infection has declined to $80 \%$, which is unacceptable for clinical practice [10-12]. 
Some studies [13-14] have pointed out that the primary eradication rate of H. Pylori can reach $90 \%$ if the appropriate antibiotics are selected according to the local antibiotics resistance of H. Pylori. Therefore, paying attention to the research and monitoring of local antibiotic resistant strains can not only understand the situation of $\mathrm{H}$. Pylori resistance, but also guide clinical medication, overcome the production of antibiotic-resistant strains and improve the radical cure rate. The resistance rate of each of the antibiotics used in the treatment of $\mathrm{H}$. Pylori infection should be determined in each geographical area to guide clinical practice.

Therefore, we performed a survey of H. Pylori antibiotic resistance from July 2017 to December 2019 in Nanjing area and analysed trend of H. Pylori antibiotics resistance over 5 years. In addition, we investigated the primary and post-treatment resistance prevalence to six antibiotics.

\section{Material And Methods}

\section{Patient Details}

A total of 1533 consecutive patients with upper abdominal complaints had endoscopy at Nanjing First Hospital, Jiangsu, from July 2017 to December 2019. 329 patients had been treated for H. pylori infection before endoscopy. Histology, rapid urease, serology testing and culture were used to determined $\mathrm{H}$. pylori status. Patients receiving antibiotics, proton-pump inhibitors, $\mathrm{H}_{2}$ receptor blockers or bismuth salts within 4 weeks of the endoscopy were excluded. Four biopsy specimens were taken from the antral mucosa and one biopsy specimen was taken from the antral body during endoscopy, two biopsies were used for histological examination, one for rapid urease test, and the other for culture and sensitivity.

\section{Culture}

For H. pylori culture, biopsy samples were homogenized and cultured on Campylobacter agar (Oxoid, Basingstoke, UK) supplemented with $5 \%$ sheep's blood (Bio-kont, Zhejiang, China) containing vancomycin, trimethoprim, polymyxin B and amphotericin B (Duly Biotech, Nanjing, China). Plates were incubated in microaerophilic conditions at $37^{\circ} \mathrm{C}$ for $2-3$ days.

\section{Antibiotic susceptibility test}

Antibiotic susceptibility of the isolated H. pylori strains to 6 antibiotics (amoxicillin, clarithromycin, levofloxacin, furazolidone, tetracycline, and metronidazole) were tested by the agar dilution method. The resistance breakpoints of each antibiotics are referred to previous reports or the Clinical and Laboratory Standards Institute (CLSI) guidelines [15], H. pylori strain ATCC43504 was included as an antibiotic susceptibility testing quality control. All antibiotic susceptibility tests were conducted at the Institute of Gastroenterology, Nanjing First Hospital, Nanjing Medical University. 


\section{Statistical analysis}

Data analysis was performed by SPSS 17.0 (IBM SPSS, Chicago, IL,USA). Frequencies and percentages were used to describe the antibiotic resistance rates of $\mathrm{H}$. pylori isolates. The univariate association between each factor was quantified by Fisher's exact test and the chi-square test. A probability value of < 0.05 from a two-tailed test was considered statistically significant.

\section{Results}

\section{Overall H. pylori antibiotic resistance}

To have some insight into the latest regional resistance of $\mathrm{H}$. pylori to the 6 most frequently used antibiotics, we performed an investigation based on $1533 \mathrm{H}$. pylori strains from July 2017 to December 2019. The resistance rates to amoxicillin, clarithromycin, levofloxacin, furazolidone, tetracycline, and metronidazole were $2.74 \%, 47.03 \%, 33.59 \%, 0.91 \%, 0.52 \%$ and $80.76 \%$, respectively.

\section{The trend of $\mathrm{H}$. Pylori antibiotics resistance over 5 years in Nanjing area}

Our preliminary article [16] showed that the resistance rates to amoxicillin, clarithromycin, levofloxacin, furazolidone, and metronidazole were $7.36 \%, 29.47 \%, 14.74 \%, 5.78 \%$, and $60.53 \%$, respectively, in May 2014 to May 2015. It was a pity that we didn't do the resistance rate to tetracycline at that time. During the past 5 years, metronidazole resistance was increased significantly from ranging from $60.53-80.76 \%$. Compared with the level of past, the resistance rate of clarithromycin, levofloxacin were significant higher, ranging from $29.47-47.03 \%, 14.74-33.59 \%$, respectively. Interestingly, compared to the past 5 years, our recent results showed lower resistance rates to amoxicillin $(P=0.000)$ and furazolidone $(P=0.000)$. Results are depicted in Table 1.

Table 1 Trends of resistance rate of $H$. Pylori to antibiotics in Nanjing

\begin{tabular}{|c|c|c|c|}
\hline \multirow[b]{2}{*}{ antibiotics } & \multicolumn{2}{|c|}{ resistance rate in different periods } & \multirow[b]{2}{*}{$P$} \\
\hline & 2014.05-2015.05 & 2017.07-2019.12 & \\
\hline amoxicillin & 14/190冈7.36\%》 & $42 / 1533 \rrbracket 2.74 \% \bigotimes$ & 0.000 \\
\hline clarithromycin & $56 / 190 \rrbracket 29.47 \% \rrbracket$ & 721/1533ه47.03\%区 & 0.000 \\
\hline levofloxacin & 28/190ه14.74\%区 & $515 / 1533 \otimes 33.59 \% \rrbracket$ & 0.000 \\
\hline furazolidone & 11/190®5.78\%》 & 14/1533凶0.91\%区 & 0.000 \\
\hline metronidazole & 115/190冈60.53\%ष & $1238 / 1533 \rrbracket 80.76 \% \rrbracket$ & 0.000 \\
\hline
\end{tabular}




\section{The primary and post-treatment resistance prevalence to six antibiotics}

The resistance rates of $\mathrm{H}$. pylori (primary Vs post-treatment) to amoxicillin, clarithromycin, levofloxacin, furazolidone, tetracycline and metronidazole were $1.83 \%$ Vs $6.08 \%, 38.62 \%$ Vs $77.81 \%, 27.41 \%$ Vs $56.23 \%, 0.58 \%$ Vs $2.13 \%, 0.33 \%$ Vs $1.22 \%, 78.57 \%$ Vs $88.75 \%$, respectively during the period of July 2017 to December 2019. Compared with the primary resistance rates, the post-treatment resistance rates to six antibiotics were higher, clarithromycin and levofloxacin were the most obvious $(P=0.000)$. Results are depicted in Table 2.

Table 2 The primary and post-treatment resistance prevalence to six antibiotics from July 2017 to December 2019

\begin{tabular}{|c|c|c|c|}
\hline \multicolumn{4}{|c|}{ the primary resistance the post-treatment resistance } \\
\hline amoxicillin & $22 / 1204 \rrbracket 1.83 \% \rrbracket$ & $20 / 329 \otimes 6.08 \% \rrbracket$ & $P$ \\
\hline clarithromycin & $465 / 1204 \llbracket 38.62 \% \rrbracket$ & $256 / 329 \llbracket 77.81 \% \rrbracket$ & 0.000 \\
\hline levofloxacin & $330 / 1204 \llbracket 27.41 \% \rrbracket$ & 185/329ه56.23\%区 & 0.000 \\
\hline furazolidone & $7 / 1204 \llbracket 0.58 \% \rrbracket$ & $7 / 329 \rrbracket 2.13 \% \rrbracket$ & 0.022 \\
\hline tetracycline & $4 / 1204 \rrbracket 0.33 \% \rrbracket$ & $4 / 329 \rrbracket 1.22 \% \rrbracket$ & 0.124 \\
\hline metronidazole & $946 / 1204 \rrbracket 78.57 \% \bigotimes$ & \ 292/329ه88.75\%区 & 0.000 \\
\hline
\end{tabular}

\section{Discussion}

H. pylori is an important human pathogen which plays a significant role in the pathogenesis of upper gastrointestinal tract diseases. Actual infection rates is nearly $50 \%$ of the world's population and varies geographically, being higher in developing countries [17-18]. Unfortunately, $\mathrm{H}$. pylori is a relatively difficult infection to treat. The gastric habitat offers sanctuaries beneath the mucous layer and within the lumen of gastric glands and pits that partially shelter $\mathrm{H}$. pylori from the topical or luminal effects of some antibiotics. Gastric acidity inactivates many other antibiotics. Furthermore, H. pylori has shown a propensity to rapidly acquire resistance to many classes of antibiotics after exposure to those agents in the form of monotherapy. These include the fluoroquinolones, the macrolides, the nitroimidazoles. For these reasons, antibiotic resistance remains the major determinant of treatment outcomes, the success rate of eradication has been seriously limited for the progressive increase in antibiotic resistance [19]. Thus, in order to determine the status of $\mathrm{H}$. pylori resistance and its patterns in patients and reliably select sensitive antibiotics for clinical use, it is necessary to perform an investigation especially in the absence of individualized $\mathrm{H}$. pylori culture and antibiotic susceptibility test in Nanjing area.

Previous studies reported that the resistance rates of $\mathrm{H}$. pylori strains collected by different geographic areas in China were $0.01 \%-6.8 \%$ to amoxicillin, $17.6 \%-37.5 \%$ to clarithromycin, $20.6 \%-33.5 \%$ to 
levofloxacin, $0.01 \%-0.1 \%$ to furazolidone, $0.01 \%-5.8 \%$ to tetracycline and $60 \%-70 \%$ to metronidazole [9, $15,20-21]$. Our current data showed that the overall resistance rates of amoxicillin, clarithromycin, levofloxacin, furazolidone, tetracycline, and metronidazole were $2.74 \%, 47.03 \%, 33.59 \%, 0.91 \%, 0.52 \%$ and $80.76 \%$, respectively. Resistance to metronidazole has been observed worldwide and presently occurs quite commonly in several countries. The $80.76 \%$ resistant rate of this study from July 2017 to December 2019 is higher than that in the past. The high resistant rate was attributable to previous treatment of gynecologic infection or to the therapy of intestinal parasitic infections, which occur frequently in our country. Our data illustrated that the resistance rate of clarithromycin was $47.03 \%$, whereas it was $29.47 \%$ in the period of May 2014 to May 2015. According to Maastricht $V$ consensus, when the resistance rate of $\mathrm{H}$. pylori to clarithromycin in a certain area exceeds $15 \%$, the triple therapy based on PPI containing clarithromycin should not be used as an empirical eradication program of $\mathrm{H}$. pylori, and clarithromycin resistance test is recommended. The resistance rate of $\mathrm{H}$. pylori to levofloxacin reported in the our study was higher than the $14.74 \%$ that was reported in the period of May 2014 to May 2015. Levofloxacin was also widely used in the treatment of urogenital system and respiratory tract infection, which may be the reason for the high resistance rate. However, the prevalence of resistance to amoxicillin, furazolidone and tetracycline were low. Besides, they were all cheap. Therefore, the three antibiotics were recommended due to a lack of resistance and low cost.

We also found that the resistance rates of $\mathrm{H}$. pylori (primary Vs post-treatment) to amoxicillin, clarithromycin, levofloxacin, furazolidone, tetracycline and metronidazole were $1.83 \%$ Vs $6.08 \%, 38.62 \%$ Vs $77.81 \%, 27.41 \%$ Vs $56.23 \%, 0.58 \%$ Vs $2.13 \%, 0.33 \%$ Vs $1.22 \%, 78.57 \%$ Vs $88.75 \%$, respectively. Compared with the primary resistance rates, the post-treatment resistance rates to six antibiotics were higher. Among them, clarithromycin, levofloxacin and metronidazole increased significantly. So it was suggested that such drugs should be carefully selected when it was not the first time a patient has been eradicated. The post-treatment resistance rates to furazolidone and tetracycline were still relatively low. Compared with the primary resistance rate, the post-treatment resistance rate to amoxicillin was higher. For post-treatment eradication of $\mathrm{H}$. pylori patients, drug sensitivity test was recommended to clarify the drug resistance of amoxicillin and guide accurate treatment.

\section{Conclusions}

Based on the current research data, the resistance rate of $\mathrm{H}$. pylori to clarithromycin, metronidazole and levofloxacin in Nanjing were high, and they were on the rise. In order to improve the eradication rate of the first treatment as much as possible, it is recommended to select the antibiotics with low resistance rate in Nanjing. For post-treatment eradication patients, clinicians should considered the regional drug resistance rate, personal antibiotic exposure history, economic benefit ratio, adverse drug reactions, drug availability and other aspects. According to the results of antibiotics sensitivity test, the combination of two sensitive antibiotics can achieve high eradication rate, reduce the incidence of adverse reactions, and reduce the secondary resistance rate. It is an effective strategy to cope with the current situation of high resistance rate of $\mathrm{H}$. pylori in Nanjing area. 


\section{Abbreviations}

H. pylori

Helicobacter pylori

\section{Declarations}

\section{Acknowledgements}

It is acknowledged that in the data collection we obtained valuable help from Helicobacter Pylori Research Key Laboratory, Nanjing Medical University.

\section{Authors' contributions}

Zongdan Jiang, Zhenyu Zhang and Shukui Wang conceived, organized and supervised the project, and proofread the manuscript. Zongdan Jiang, Dan Ran and Bangshun He collected and analysed the data, and drafted the manuscript. Zhibing Wang supervised statistical analysis. Zongdan Jiang, Dan Ran and Bangshun He completed H.pylory laboratory identification and antimicrobial sensitity testing. All authors approved the final version of the manuscript.

\section{Funding}

This study was supported by grants from Special funds for key research and development plans of Jiangsu Province in 2019 (NO. BE2019614).

\section{Availability of data and materials}

The datasets used and/or analysed during the current study are available from the corresponding author on reasonable request.

\section{Ethics approval and consent to participate}

Not applicable.

\section{Consent for publication}

Not applicable.

\section{Competing interests}

The authors declare that they have no competing interests.

\section{References}


1. Moayyedi P, Hunt RH. Helicobacter pylori public health implications. Helicobacter. 2004; 9 (Suppl 1): 67-72. doi:10.1111/j.1083-4389.2004.00250.x.

2. Duck WM, Sobel J, Pruckler JM, Song Q, Swerdlow D, Friedman C, SulkaA, Swaminathan B, Taylor T, Hoekstra M, Griffin P, Smoot D, Peek R, Metz DC, Bloom PB, Goldschmidt S, Parsonnet J, Triadafilopoulos G, PerezPerez GI, Vakil N, Ernst P, Czinn S, Dunne D, Gold BD. Antimicrobial resistance incidence and risk factors among Helicobacter pylori-infected persons, United States. Emerg Infect Dis. 2004;10(6):1088-94. doi:10.3201/eid1006.030744.

3. Malfertheiner P, Chan FK, McColl KE. Peptic ulcer disease. Lancet. 2009; 374(9699):1449-61. doi: 10.1016/S0140-6736(09)60938-7.

4. Moore JM, Salama NR. Mutational analysis of metronidazole resistance in Helicobacter pylori. Antimicrob Agents Chemother. 2005;49(3):1236-7. doi: 10.1128/AAC.49.3.1236-1237.2005 .

5. Malfertheiner P, Megraud F, O'Morain CA, Atherton J, Axon AT, Bazzoli F, Gensini GF, Gisbert JP, Graham DY, Rokkas T, El-Omar EM, Kuipers EJ. Management of Helicobacter pylori infection-the Maastricht IV/Florence Consensus Report. Gut. 2012;61(5):646-664. doi: 10.1136/gutjnl-2012302084.

6. Xie C, Lu NH. Review: clinical management of Helicobacter pylori infection in China. Helicobacter. 2015;20 (1):1-10. doi: 10.1111/hel.12178.

7. Hu Y, Zhu Y, Lu NH. Primary antibiotic resistance of Helicobacter pylori in China. Dig Dis Sci. 2017; 62 (5): 1146-1154. doi.:10.1007/s10620-017-4536-8.

8. Liu WZ, Xie Y, Lu H, Cheng H, Zeng ZR, Zhou LY, Chen Y, Wang JB, Du YQ, Lu NH, on behalf of Chinese Society of Gastroenterology, Chinese Study Group on Helicobacter pylori and Peptic Ulcer. 2018. Fifth Chinese National Consensus Report on the management of Helicobacter pylori infection. Helicobacter. 2018;23(2):e12475. doi: 10.1111/hel.12475..

9. Su P, Li Y, Li H, Zhang J, Lin L, Wang Q, Guo F, Ji Z, Mao J, Tang W, Shi Z, Shao W, Mao J, Zhu X, Zhang $X$, Tong Y, Tu H, Jiang M, Wang Z, Jin F, Yang N, Zhang J. Antibiotic resistance of Helicobacter pylori isolated in the southeast coastal region of China. Helicobacter. 2013; 18(4):274-9. doi: 10.1111/hel.12046.

10. Graham DY, Fischbach L. Helicobacter pylori treatment in the era of increasing antibiotic resistance. Gut. 2010;59(8):1143-53. doi: 10.1136/gut.2009.192757..

11. Zheng Q, Chen WJ, Lu H, Sun QJ, Xiao SD. Comparison of efficacy of triple versus quadruple therapy on eradication of Helicobacter pylori and antibiotic resistance. J Dig Dis. 2010;11(5):313-8. doi: 10.1111/j.1751-2980.2010.00457.x.

12. Malfertheiner $P$, Bazzoli F, Delchier JC, Celiñski K, Giguère $M$, Rivière $M$, Mégraud F, Pylera Study Group. Helicobacter pylori eradication with a capsule containing bismuth subcitrate potassium, metronidazole, and tetracycline given with omeprazole versus clarithromycin-based triple therapy: a randomized, open-label, non-inferiority, phase 3 trial. Lancet. 2011; 377(9769):905-913. doi:10.1016/S0140-6736(11)60020-2. 
13. Graham DY, Rimbara E. Understanding and appreciating sequential therapy for Helicobacter pylori eradication. J Clin Gastroenterol. 2011; 45 (4): 309-313. doi: 10.1097/MCG.0b013e31820ac05e.

14. Rimbara E, Fischbach LA, Graham DY. Optimal therapy for Helicobacter pylori infections. Nat Rev Gastroenterol Hepatol. 2011; 8 (2): 79-88. doi: 10.1038/nrgastro.2010.210.

15. Zhang YX, Zhou LY, Song ZQ, Zhang JZ, He LH, Ding Y. Primary antibiotic resistance of Helicobacter pylori strains isolated from patients with dyspeptic symptoms in Beijing: a prospective serial study. World J Gastroenterol. 2015; 21(9):2786-2792. doi: 10.3748/wjg.v21.i9.2786.

16. Shi XF, Jiang ZD, Zhang ZY, Wang ZB, Wang WH, Lin HH, Xu X, Zhang YS, Zhao XY. Research on the infection and medicine-resistance of Helicobacter pylori in Jiangsu Province Nanjing District. Chin J Clin Gastroenterol, 2016;28(4):206-208. Doi:10.3870/Icxh.j.issn.1005-541X.

17. Correa P, Piazuelo MB. Natural history of Helicobacter pylori infection. Dig Liver Dis. 2008;40(7):4906. doi: 10.1016/j.dld.2008.02.035.

18. Khan A, Farooqui A, Raza Y, Rasheed F, Manzoor H, Akhtar SS, Quraishy MS, Rubino S, Kazmi SU, Paglietti B. Prevalence, diversity and disease association of Helicobacter pylori in dyspeptic patients from Pakistan. J Infect Dev Ctries. 2013;7(3):220-8. doi: 10.3855/jidc.2942.

19. Thung I, Aramin H, Vavinskaya V, Gupta S, Park JY, Crowe SE, Valasek MA. Review article: the global emergence of Helicobacter pylori antibiotic resistance. Aliment Pharmacol Ther. 2016; 43(4):514-533. doi: 10.1111/apt.13497.

20. Song Z, Zhang J, He L, Chen M, Hou X, Li Z, Zhou L. Prospective multi- region study on primary antibiotic resistance of Helicobacter pylori strains isolated from Chinese patients. Dig Liver Dis. 2014; 46(12):1077-1081. doi: 10.1016/j.dld.2014.08.038.

21. Ji Z, Han F, Meng F, Tu M, Yang N, Zhang J. The Association of age and antibiotic resistance of Helicobacter Pylori: a study in Jiaxing City, Zhejiang province China. Medicine (Baltimore). 2016;95(8):e2831. doi:10.1097/MD.0000000000002831. 